\title{
Follow-up of Breast Cancer Patients in Ghana: Challenges to Community-based Surveillance
}

\author{
Dennis O. Laryea*1, 2, Baffour Awuah", ${ }^{1,}$, Yaw A. Amoako ${ }^{1,2}$, Samuel Mensah ${ }^{1}$ and Fred K. \\ Awittor $^{2}$
}

${ }^{1}$ Public Health, Komfo Anokye Teaching Hospital, Kumasi, Ghana; ${ }^{2}$ Kumasi Cancer Registry, Kumasi, Ghana

\section{Objective}

To identify challenges to community-based surveillance and follow up of breast cancer cases in Ghana

\section{Introduction}

Cancers are among the leading causes of deaths globally. In subSaharan Africa, cancer-related deaths have been projected to increase significantly in the next few decades. Information on cancer is essential in planning and implementing cancer control and prevention activities. Registration and follow-up of cancer cases to estimate survival are useful tools in cancer control programmes. In Ghana, despite the existence of a national cancer prevention and control strategy, not much attention has been given to the problem. Cancer survival has been found to be poor in most developing countries due to late reporting. While late reporting may be a significant factor in cancer survival, the ability of clinical and community health staff to follow-up on cases can help and provide accurate information on cancer survival.

\section{Methods}

We set out to follow-up 136 breast cancer cases diagnosed from 2006 to 2008 among residents in the city of Kumasi. We reviewed the case notes to determine their places of residence and other relevant demographic information. Cases were contacted via telephone calls or visits to the stated residential addresses. The status of the case on contact (dead or alive) was noted.

\section{Results}

A total of 51(37.5\%) out of the 136 cases could be contacted either personally or by contact with relations where death had occurred. Forty one of those contacted were by phone (41) and the remaining (10) by residential address. All remaining $85(62.5 \%)$ cases could not be contacted by telephone. Twenty six of these contacts did not have a phone number indicated in their folders. Of the remaining 59 cases with phone numbers in the folders, attempts to call the numbers resulted in the following responses: 'wrong phone numbers' $(n=16)$, 'phone number switched off' $(n=13)$ or 'number not reachable' $(\mathrm{n}=30)$. Examination of clinical records for the residential addresses, of all 85 cases who could not be reached by phone revealed 3 cases with no residential addresses 21 with incomplete addresses, 33 with addresses that could not be located and 28 were unknown to current occupants of the residential address given. One (1) case had neither an address nor a telephone number indicated in the folder. Among those for whom contact was established, 23 representing $45.1 \%$ were alive at the time of contact.

\section{Conclusions}

This study has highlighted challenges associated with following up patients in low resourced settings such as Ghana. Such situations may present a public health risk in cases of communicable disease. There is the need to ensure that demographic data captured for patients are verified in order to allow for easy tracing when necessary. While mobile telephony may be useful in surveillance, multiple mobile phone numbers including those of relatives may facilitate easier follow-up of patients. There is a general need to ensure a more robust addressing system in Ghana to ensure that residences can be easily traced.

\section{Keywords}

cancer; follow-up; noncommunicable disease surveillance

\section{Acknowledgments}

We wish to acknowledge the support of the African Cancer Registry Network (AFRCN), the Komfo Anokye Teaching Hospital and Registrars at the Kumasi Cancer Registry

\section{References}

Bah E, Carrieri MP, Hainaut P, Bah Y, Nyan O, Taal M. 20-Years of Population-Based Cancer Registration in Hepatitis B and Liver Cancer Prevention in The Gambia, West Africa. PLoS One [Internet]. 2013 Sep 30 [cited 2013 Dec 4];8(9). Available from: http://www.ncbi.nlm. nih.gov/pmc/articles/PMC3787012/

Bosu WK. A Comprehensive Review of the Policy and Programmatic Response to Chronic Non-Communicable Disease in Ghana. Ghana Med J. 2012 Jun; 46(2 Suppl):69-78.

Hadji M, Nahvijou A, Seddighi Z, Beiki O, Mohagheghi MA, MosaviJarrahi A, et al. Challenges to promoting population-based cancer registration in iran: a workshop report. Asian Pac J Cancer Prev APJCP. 2013;14(10):6189-93.

Hanna TP, Kangolle AC. Cancer control in developing countries: using health data and health services research to measure and improve access, quality and efficiency. BMC Int Heal Hum Rights. 2010 Oct $13 ; 10: 24$.

O'Brien KS, Soliman AS, Awuah B, Jiggae E, Osei-Bonsu E, Quayson $\mathrm{S}$, et al. Establishing effective registration systems in resource-limited settings: cancer registration in Kumasi, Ghana. J Regist Manag. 2013; 40(2):70-7.

Laryea DO, Awuah B, Amoako YA, Osei-Bonsu E, Dogbe J, LarsenReindorf R, et al. Cancer incidence in Ghana, 2012: evidence from a population-based cancer registry. BMC Cancer. 2014 May 23; 14(1):362.

*Dennis O. Laryea

E-mail: dlaryea@kathhsp.org 